\title{
Proposal of a Wireless Power Transfer Technique for Low-power Multi-receiver Applications
}

\author{
Yinggang $\mathrm{Bu}^{1}$, Tsutomu Mizuno ${ }^{1}$ and Hidetaka Fujisawa ${ }^{2}$ \\ ${ }^{1}$ Faculty of Engineering, Shinshu University, Nagano 380-8553, Japan \\ ${ }^{2}$ Optoelectronics Co., Ltd., Warabi, Saitama 335-0002, Japan
}

In this paper, we proposed and verified the feasibility of a unique wireless power transfer structure called a rail transformer to drive multiple low-power devices such as electronic shelf label (ESL) devices. The rail transformer is composed of a rectangular, circular-shaped transmitting yoke and two transmitting coils to provide wireless power. Multiple receiving yokes coupled with receiving coils are installed across the elongated edge of the transmitting yoke. It can be driven by low-frequency $\mathrm{AC}$ power at $50 / 60$ Hz. In our prototype, the transmitting yoke is $900 \mathrm{~mm}$ long and $15 \mathrm{~mm}$ wide. We obtained the minimal induced wireless power, and the voltage was about $61 \mathrm{~mW}$ and $3.5 \mathrm{~V}$, which is sufficient to drive a typical ESL device. By designing a non-uniform gap thickness between the transmitting and receiving yokes at the specific locations, we improved the uniformity of the induced power for multiple ESL devices.

Index Terms - wireless power transfer, multi-receiver applications, electronic shelf label, rail transformer.

\section{INTRODUCTION}

$\mathbf{R}^{\mathrm{E}}$ ECENTLY, the research of the wireless power transfer (WPT) technology has attracted broad interests because of its convenience of connectivity and operational safety [1-3]. Unlike conventional electrical power supply methods, where direct electrical contact is necessary, WPT uses the electromagnetic induction phenomenon to transfer electronic power without contact. Since there is no metal contact directly exposed to a user, safety and durability are increased. This technology has been used in some power transfer devices such as electric toothbrushes, electric vehicles, and televisions [2-3]. In these applications, wireless power transmission is realized by a pair of coils: one for transmitting power in the charger and another for receiving power in the charged device. In these schemes, the power transmission efficiency is highly dependent on the mutual positions of the transmitting and receiving coils. There is only a limited tolerance for generating efficient induction between the two coils. Hence, mechanical guidance is generally designed into the receiving coil to aid a user in matching its position to the transmitting coil.

Another scheme is to use a single transmitting coil to induce multiple receiving coils [4-5]. One example is the provision of electrical power supply to an electronic shelf label (ESL), which is an electrical tag installed on a shelf to display the sales information for the articles [6-7]. As shown in Fig. 1, ESL is quite a convenient media for displaying price information in a large-scale mall. This is not only because frequent price changes to hundreds or thousands of paper labels bring a heavy labor burden but also because frequent price changes to paper labels introduce a price update delay between the point of sale (POS) machine and the local price at the shelf. As a result, this delay causes troublesome transactions because of the price mismatch. However, because an ESL is always connected to the database of the POS

Manuscript received March 20, 2015. Corresponding author: Y.Bu (email: buyinggang@shinshu-u.ac.jp).

Digital Object Identifier inserted by IEEE

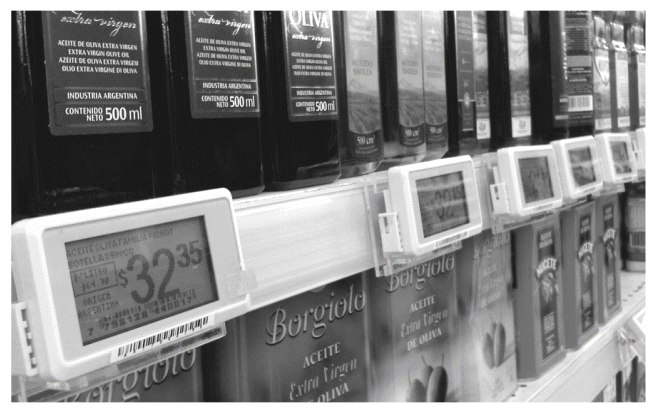

Fig. 1. A sample photograph of the ESL system. (By courtesy of Optoelectronics Co., Ltd. )

machines, price information is updated at the same time. It becomes much easier to realize dynamic and efficient price management compared with conventional paper media. Like paper media price labels, there are multiple ESL devices on the shelves and they can be freely positioned as long as their locations correspond to the articles.

A popular ESL device design comprises a LCD (liquid crystal display) for displaying the price, a wireless communication unit for receiving the latest price information from the POS database, and button battery/batteries as power supply. However, since a button battery has relatively low power capacity, it is difficult for an ESL device to have a display that has high contrast as a conventional paper label. Another disadvantage of the current ESL devices is that after several years, a large number of batteries will simultaneously be disposed, which will potentially cause environmental pollution. To reinstall new batteries in tens of thousands of ESL devices is also a heavy labor burden for a large-scale mall. To solve such problems, we proposed a wireless power transmission method as the power supply for an ESL device. The basic thinking was to install a transmitting coil on the shelf and a receiving coil in each individual ESL device. One might think of the scheme to use electric coil magnetic resonance working at several thousands of $\mathrm{Hz}$ to increase the coupling efficiency like a converter [4], [8]. However, in mall 


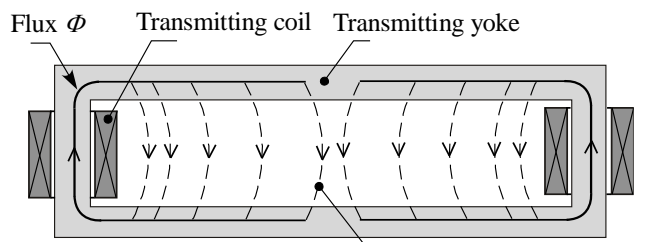

(a) Leakage flux $\Phi_{l}$

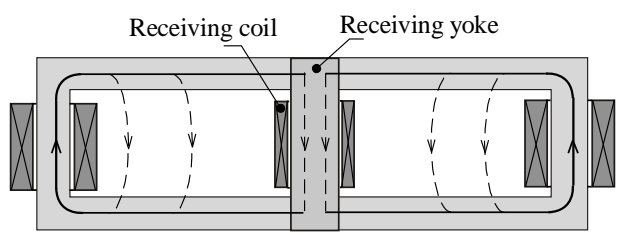

(b)

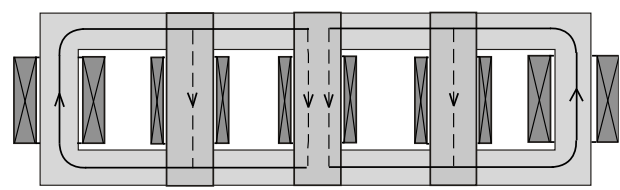

(c)

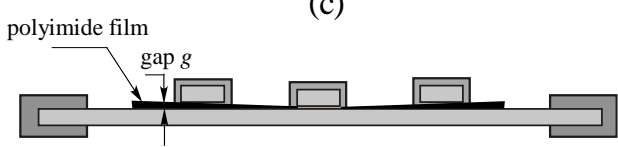

(d)

Fig. 2. Basic structure of the proposed wireless power transmission structure. (a) plane view without receiving yoke. (b) plane view with one receiving yoke. (c) plane view with three receiving yokes (d) side view.

applications, there needs to be a high power supply working at high frequency at each shelf. In addition to the installation cost, it is practically difficult to shield the electromagnetic radiation from such power supplies. In this paper, we propose a unique transmitting coil structure suitable for wirelessly feeding multiple ESL devices directly from a $50 / 60 \mathrm{~Hz}$ electrical power line. Since this wireless power structure is similar to those used on railways, we call it a "rail transformer." We analyzed the principles of the proposed wireless power transmission scheme and optimized the magnetic flux distribution by designing a specific gap. Finally, we verified the feasibility of driving multiple ESL devices through the experiments.

\section{BASIC STRUCTURE AND WORKING PRINCIPLE}

\section{A. Basic structure and working principle}

Figure 2 shows the basic structure of the proposed scheme. The base for transmitting wireless power is an elongated, rectangular, circle-shaped yoke as the transmitting yoke (Fig. 2(a)). A pair of transmitting coils surrounds the short edges of the transmitting yoke as the electrical power supply. When the transmitting coils are supplied with AC current, the coils induce magnetic flux, which becomes the leakage flux distributed between the elongated edges of the transmitting yoke. As shown in Fig. 2(b), if we place a receiving yoke across the elongated edges of the transmitting yoke, it will form an enclosed magnetic loop. As the result, the flux flows through the receiving yoke.

The winding of a coil around this receiving yoke will further
TABLE I

SPECIFICATIONS OF PROTOTYPE

\begin{tabular}{llcc}
\hline \hline \multicolumn{1}{c}{ Item } & & Value & Unit \\
\hline Transmitting & Diameter of conductor & 0.12 & $\mathrm{~mm}$ \\
coil & Number of turns & 5000 & turn \\
& DC resistance of coil & 110 & $\Omega$ \\
Receiving & Diameter of conductor & 0.16 & $\mathrm{~mm}$ \\
coil & Number of turns & 1200 & turn \\
& DC resistance of coil & 50 & $\Omega$ \\
Transmitting & Material & Silicon lamination & - \\
yoke & Thickness & 0.35 & $\mathrm{~mm}$ \\
& Flux density & $>1.64$ & $\mathrm{~T}$ \\
\hline
\end{tabular}

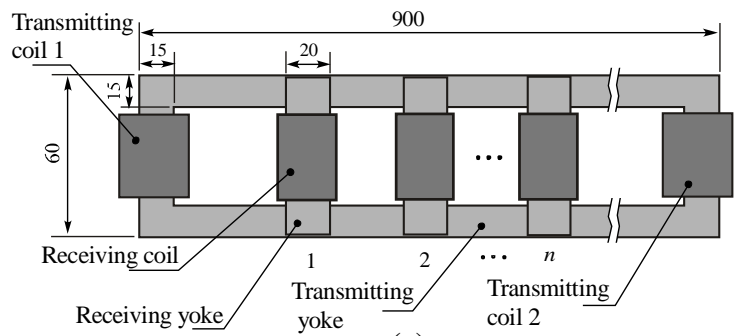

(a)

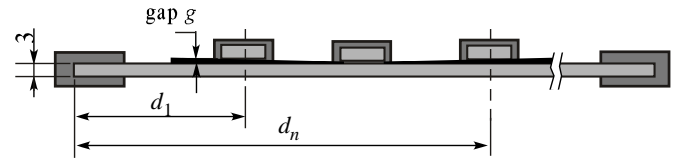

(b)

Fig. 3. Basic structure of prototype (a) plane view (b) side view (unit: mm).

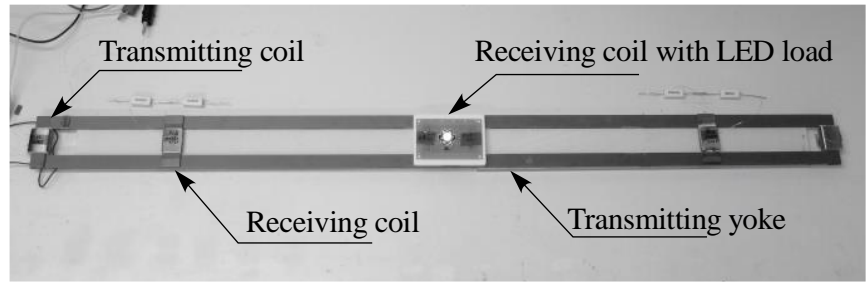

Fig. 4. Photograph of the prototype device for wireless power transmission.

generate an induced voltage in the coil. Hence, if we install such an elongated transmitting yoke and transmitting coils on the shelf and install such a receiving yoke and receiving coil into an ESL device, the ESL will be powered without the use of batteries. If the power supply is from an AC power line, we do not need to worry about power consumption in the ESL device and can further install a high-contrast LCD in the ESL device to enhance the contrast to a level comparable to that of paper media. In any case, the replacement of batteries is not necessary.

We now analyze the situation where multiple receiving yokes (i.e., multiple ESL devices) are installed across the transmitting yoke. As illustrated in Fig. 2 (c), multiple receiving yokes are installed across the elongated edges of the transmitting yoke and they can be slid along the elongated edges. When the transmitting coils are powered by an AC power line, the flux will be redistributing at each receiving yoke. As we know from electromagnetic induction theory, the 
flux in each receiving yoke is not equal. The closer we get to the transmitting coils, the larger the flux becomes. This creates a problem for the application of ESL, where each ESL at a different location might be powered at a different level. This is similar to the structure of a railway but with limited length. The elongated edges of the transmitting yoke are like the tracks, and the multiple receiving yokes are like railroad ties across the tracks. When the transmitting coils are powered by an $\mathrm{AC}$ power line, they work similar to a transformer. For convenience, we refer to the entire structure as the rail transformer.

To solve this problem, we designed polyimide films as thin gap layers on the surface of the elongated edges of the transmitting yoke. To compensate for the distribution of the leakage flux, the polyimide film is designed at different thicknesses corresponding to the distance to the transmitting coils, as shown in Fig. 2(d). The closer we get to the transmitting coils, the greater the gap thickness becomes. A thicker gap has greater magnetic reluctance between the transmitting and receiving yokes. Hence, in theory, by properly designing the gap thickness, we can make the flux flowing to each receiving yoke equal. This means that each ESL installed at any location will be supplied with the same power.

\section{B. Experimental prototype design}

Based on our analysis, we designed a prototype, as shown in Fig. 3. The transmitting yoke had an elongated edge of $\mathrm{L}=$ $900 \mathrm{~mm}$ and a short edge of $\mathrm{W}=60 \mathrm{~mm}$. This is a typical dimension of a shelf edge, where ESL devices are installed. The transmitting yoke was composed of a magnetic steel plate with a width of $15 \mathrm{~mm}$ and a thickness of $3 \mathrm{~mm}$, with a lamination layer covering its surface. In the experiment, we installed several receiving yokes along the elongated edge of the transmitting yoke. Each receiving yoke was enclosed by a receiving coil with 1200 turns. The specifications of the prototype are shown in Table. I. To simulate a practical ESL device, we connected a load of $200 \mathrm{ohms}$ resistance to each receiving coil. Two transmitting coils were installed at the short edges of the transmitting yoke and connected to an $\mathrm{AC}$ power supply in parallel at $80 \mathrm{~V} / 60 \mathrm{~Hz}$. Fig. 4 shows a photograph of the prototype where the receiving coils were also connected to an LED indicator to indicate that it was working.

\section{EXPERIMENTAL EVALUATION}

In this section, we first investigated the flux density distribution by opening the receiving yoke and investigated the power transmission characteristics by connecting a load to the receiving coil.

\section{A. Single receiving yoke installed}

First, we supplied AC power at $80 \mathrm{~V} / 60 \mathrm{~Hz}$ to the transmitting coils and measured the magnetic flux density in the transmitting yoke without installing receiving yokes, as shown in Fig. 5. Curve $B$ of the transmitting yoke in Fig. 5 shows the flux density distribution without any receiving yoke.

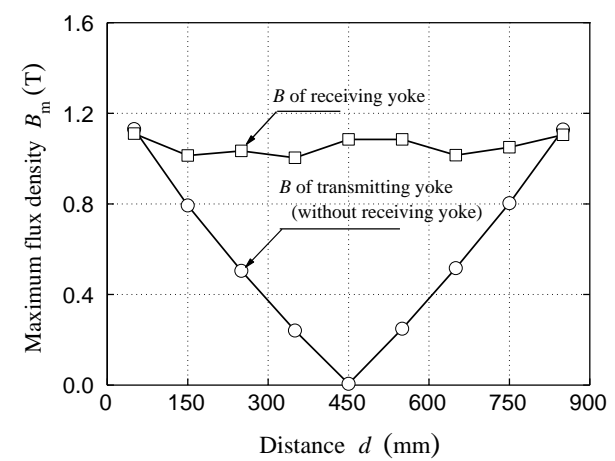

Fig. 5. Maximum flux density of the transmitting yoke with one receiving yoke.

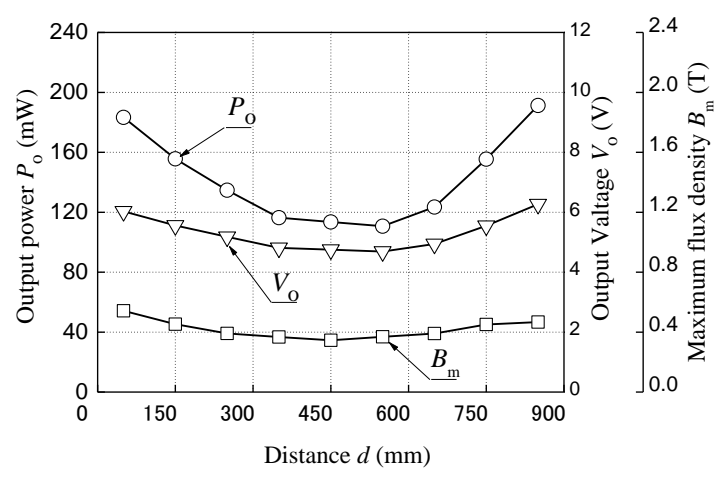

Fig. 6. Characteristics of power transmission with one receiving coil.

The maximum flux density $B_{\mathrm{m}}$ was measured using a search coil. At the location of $50 \mathrm{~mm}$ and $850 \mathrm{~mm}$, in the area closest to the transmitting coils, the magnetic flux density is at the peak value of $1.13 \mathrm{~T}$. On the other hand, at the location of 450 $\mathrm{mm}$, at the central area of the elongated edge of the transmitting yoke, the flux density value reduced to almost $0 \mathrm{~T}$. As shown in Fig. 2(a), it is because the magnetic flux generated by each transmitting coil turned back and formed a magnetic energy minimum in this area.

Then, we installed one receiving yoke and slid it along the transmitting yoke from the location of one transmitting coil to another. In the experiment, we first opened the receiving coil to measure flux density. By varying the location of the yoke, we found that the density distribution became relatively uniform, as shown in the curve " $\mathrm{B}$ of receiving yoke." This is because since flux flows to the receiving yoke, the total leakage flux is reduced.

We connected a 200 -ohm resistance load to the receiving coil and investigated the power transmission characteristics. The result is shown in Fig. 6. The left y-axis is the value of the output power and the right $y$-axis is the maximum flux density. Compared with the flux density shown in Fig. 5, where the receiving coil was open, the flux density was at lower level. This is because the induced current in the receiving coil generated an inverse magnetic flux to bring down the supplied flux density. As indicated in Fig. 6, the minimal induced power is about $110 \mathrm{~mW}$ at $4.7 \mathrm{~V}$, which is enough to drive a single ESL device with a TFT (thin-film-transistor) LCD. 


\section{B. Multiple receiving yokes installed}

In practical applications, we always install multiple ESL devices on a shelf. To simulate this, we installed three receiving yokes at distances of $150 \mathrm{~mm}, 450 \mathrm{~mm}$, and $750 \mathrm{~mm}$. The measured flux density when the receiving coils were loaded is shown by a dashed line in Fig. 7. In the case where receiving yokes were placed at the transmitting yoke without gap, the measured flux density in each receiving yoke was $0.531 \mathrm{~T}, 0.360 \mathrm{~T}$, and $0.526 \mathrm{~T}$. The minimum flux was at the location of $450 \mathrm{~mm}$, which was only $68 \%$ of the flux density of the other two yokes.

Then we inserted the polyimide films under the two external receiving coils as a gap except one located at $450 \mathrm{~mm}$. In the experiment, we always fixed the central receiving coil at the location of $450 \mathrm{~mm}$, but changed the locations of these two external receiving coils. To make the flux density distribution flat, we also adjusted the polyimide thickness at each location. The experiment result is shown in the solid curve in Fig. 7. It is obvious that the gap significantly improved the flux density distribution. When these two external coils are at the distance of $150 \mathrm{~mm}$ and $750 \mathrm{~mm}$, the flat flux density distribution needs the gap of $0.55 \mathrm{~mm}$. At the distance of $250 \mathrm{~mm}$ and $650 \mathrm{~mm}$, the gap is $0.4 \mathrm{~mm}$. At the distance of $350 \mathrm{~mm}$ and $550 \mathrm{~mm}$, the gap is $0.2 \mathrm{~mm}$. The curve beneath the figure showed the thickness change to obtain the flat flux density. The maximum difference between the three yokes decreased to $3.1 \%$.

Then we connected the loads to each receiving coils and measured power transmission characteristic again at the condition with / without gap. The result is shown in Fig. 8. At the condition without gap, the input power of the rail transformer is $1.11 \mathrm{~W}$ and the output powers at each receiving coil (the dash curve with the annotation of "without gap" in Fig. 8 ) are $130 \mathrm{~mW}, 59 \mathrm{~mW}$ and $127 \mathrm{~mW}$ corresponding to the locations of $150 \mathrm{~mm}, 450 \mathrm{~mm}$ and $750 \mathrm{~mm}$. The maximal power difference is $71 \mathrm{~mW}$. The corresponding induced voltages at these receiving coils are $5.1 \mathrm{~V}, 3.4 \mathrm{~V}$ and $5 \mathrm{~V}$ respectively. The maximal voltages difference is $1.7 \mathrm{~V}$.

Then we inserted the same polyimide films under the external receiving coils at each specific location (the solid curve with the annotation of "with gap" in Fig.8). In the experiment, the input power of the rail transformer is $1.24 \mathrm{~W}$ and the output powers distribution significantly improved to be flat with up to $61 \mathrm{~mW}$. Compared to $71 \mathrm{~mW}$ power differences without gaps, this difference dropped to only $3.5 \mathrm{~mW}$. The output voltages distribution also improved to be flat at the level of $3.5 \mathrm{~V}$, and the voltages difference dropped to only $0.12 \mathrm{~V}$.

\section{CONCLUSIONS}

In this paper, we proposed a rail transformer suitable for wirelessly providing electric power to low-consumption devices, for example to an ESL device. We used a single transmitting yoke to drive multiple receiving coils, where each receiving coil served as the power supply to an ESL device. By designing gaps between the transmitting and receiving yokes at the proper locations, we verified that it is possible to

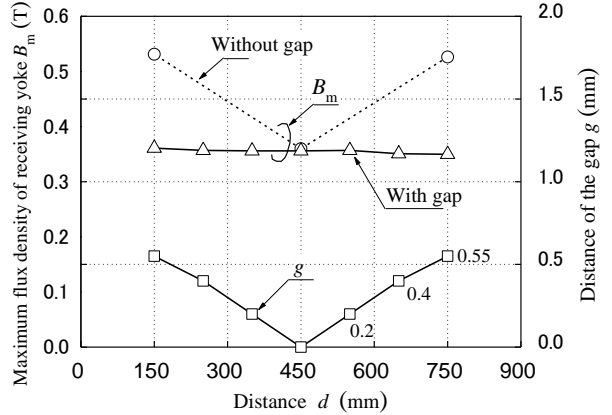

Fig. 7. Maximum flux density with three receiving yoke.

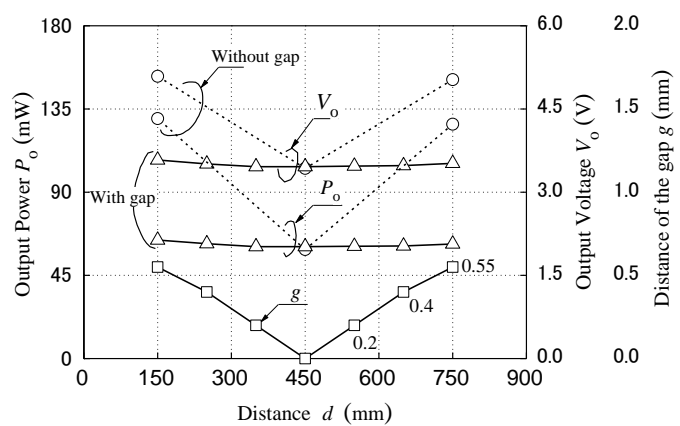

Fig. 8. Characteristics of power transmission with three receiving coils.

provide relatively uniform induced power to each ESL device. In the experiment, the power source was an AC power supply at $80 \mathrm{~V} / 60 \mathrm{~Hz}$. By driving three receiving coils, we obtained a minimum induced wireless power of up to $61 \mathrm{~mW}$, and an induced voltage of up to $3.5 \mathrm{~V}$, which is sufficient for driving a typical LCD used in an ESL device.

\section{REFERENCES}

[1] Z. Yan, Y. Li, C. Zhang, and Q. Yang, "Influence factors analysis and improvement method on efficiency of wireless power transfer via coupled magnetic resonance," IEEE Trans. Magn., vol. 50, no. 4, 2014.

[2] H. Moon, S. Kim, H. H. Park, and S. Ahn, "Design of a resonant reactive shield with double coils and a phase shifter for wireless charging of electric vehicles," IEEE Trans. Magn., vol. 51, no. 3, 2015.

[3] K. Jinwook, S. Hyeon-Chang, K. Do-Hyeon, and P. Young-Jin, "Optimal design of a wireless power transfer system with multiple selfresonators for an LED TV," IEEE Trans. Consumer Electronics, vol. 58, pp. 775-780, 2012.

[4] B. L. Cannon, J. F. Hoburg, D. D. Stancil, and S. C. Goldstein, "Magnetic Resonant Coupling As a Potential Means for Wireless Power Transfer to Multiple Small Receiver," IEEE Trans. Power Electronics, vol. 24, pp. 1819-1825, 2009.

[5] L. Chi Kwan, W. X. Zhong, and S. Y. R. Hui, "Effects of Magnetic Coupling of Nonadjacent Resonators on Wireless Power DominoResonator Systems," IEEE Trans. Power Electronics, vol. 27, pp. 19051916, 2012.

[6] H. Hong, R. Yi, T. Ricai, and X. Liyi, "Electronic Shelf Label System based on public illuminating network," in Circuits and Systems, 2008. APCCAS 2008. IEEE Asia Pacific Conference on, pp. 1103-1106, 2008.

[7] W. Ying and H. Yu, "Design of electronic shelf label systems based on ZigBee," in Software Engineering and Service Science (ICSESS), 2013 4th IEEE International Conference on, pp. 415-418, 2013.

[8] Y. Kaneko, S. Matsushita, Y. Oikawa and S. Abe, "Moving Pick-Up Type Contactless Power Transfer Systems and their Efficiency Using Series and Parallel Resonant Capacitors," IEEJ Trans. IA, vol. 128, No.7, 2008 